\title{
Ulceration is Present
}

National Cancer Institute

\section{Source}

National Cancer Institute. Ulceration is Present. NCI Thesaurus. Code C160707.

A result that ulceration is present 